\title{
Brain Modularity in Arthropods: Individual Neurons That Support "What" But Not "Where" Memories
}

\author{
Julieta Sztarker ${ }^{1,2}$ and Daniel Tomsic ${ }^{1}$ \\ ${ }^{1}$ Laboratorio de Neurobiología de la Memoria, Departamento Fisiología, Biología Molecular y Celular, Facultad de Ciencias Exactas y Naturales, \\ Universidad de Buenos Aires, Instituto de Fisiologia, Biologia Molecular y Neurociencias, Consejo Nacional de Investigaciones Científicas y Técnicas, \\ Pabellón 2 Ciudad Universitaria, Intendente Güiraldes 2160, Buenos Aires 1428, Argentina, and 2Departamento Biodiversidad y Biología Evolutiva, \\ Facultad de Ciencias Exactas y Naturales, Pabellón 2 Ciudad Universitaria, Ing. Güiraldes 2160, Universidad de Buenos Aires, Buenos Aires 1428, Argentina
}

Experiments with insects and crabs have demonstrated their remarkable capacity to learn and memorize complex visual features (Giurfa et al., 2001; Pedreira and Maldonado, 2003; Chittka and Niven, 2009). Such abilities are thought to require modular brain processing similar to that occurring in vertebrates (Menzel and Giurfa, 2001). Yet, physiological evidence for this type of functioning in the small brains of arthropods is still scarce (Liu et al., 1999, 2006; Menzel and Giurfa, 2001). In the crab Chasmagnathus granulatus, the learning rate as well as the long-term memory of a visual stimulus has been found to be reflected in the performance of identified lobula giant neurons (LGs) (Tomsic et al., 2003). The memory can only be evoked in the training context, indicating that animals store two components of the learned experience, one related to the visual stimulus and one related to the visual context (Tomsic et al., 1998; Hermitte et al., 1999). By performing intracellular recordings in the intact animal, we show that the ability of crabs to generalize the learned stimulus into new space positions and to distinguish it from a similar but unlearned stimulus, two of the main attributes of stimulus memory, is reflected by the performance of the LGs. Conversely, we found that LGs do not support the visual context memory component. Our results provide physiological evidence that the memory traces regarding "what" and "where" are stored separately in the arthropod brain.

\section{Introduction}

Historically, arthropod behavior has been considered a collection of simple, automata-like routines commanded by domainspecific brain modules working independently. However, it is now evident that the cognitive abilities of these animals are far more complex than originally assumed (Giurfa et al., 1996, 2001; Tibbetts and Dale, 2004; Chittka and Niven, 2009). In fact, Menzel and Giurfa (2001) recognized that to explain some of the complex behaviors seen in honeybees it was necessary to assume a modular organization of the brain where horizontal integration of diverse modules takes place in central sites. This organization would account for the ability of arthropods to perform new adaptive behaviors like displaying diverse types of generalization of learned stimuli and storing context-specific memories. Although there is some evidence supporting a modular organization of the brain in insects (Mizunami et al., 1998; Liu et al., 1999, 2006; Menzel, 2001), corroboration by electrophysiological studies in single neurons is missing.

Received Nov. 18, 2010; revised March 15, 2011; accepted March 16, 2011.

Author contributions: J.S. and D.T. designed research; J.S. performed research; J.S. analyzed data; J.S. and D.T. wrote the paper.

This work was supported by the following research grants to D.T.: Universidad de Buenos Aires, Grant No. X221; and Agencia Nacional de Promoción Científica y Tecnológica, Grant No. PICT 1189.

Correspondence should be addressed to Daniel Tomsic, Laboratorio de Neurobiología de la Memoria, Departamento Fisiología, Biología Moleculary Celular, Facultad de Ciencias Exactas y Naturales, Universidad de Buenos Aires, Intendente Güiraldes 2160, Pabellón II, Ciudad Universitaria, Buenos Aires 1428, Argentina. E-mail: tomsic@fbmc.fcen.uba.ar.

DOI:10.1523/JNEUROSCI.6029-10.2011

Copyright $\odot 2011$ the authors $\quad 0270-6474 / 11 / 318175-06 \$ 15.00 / 0$
The semiterrestrial crab Chasmagnathus granulatus is preyed upon by gulls; hence, an object moving overhead elicits the animal's escape. In the laboratory, a spaced training using a visual danger stimulus (VDS) moving over the crab causes a learninginduced reduction of the escape response that is retained for several days (Hermitte et al., 1999; Pedreira and Maldonado, 2003). Although at the beginning we termed this phenomenon "habituation" (Lozada et al., 1990; Tomsic et al., 1993), later investigations demonstrated that it was a more complex form of memory. The long-term modification of the escape response is exhibited only if the animal is tested in the same environment where it was trained (Tomsic et al., 1998). This and other results (Pedreira et al., 2002; Pedreira and Maldonado, 2003) indicate that the memory produced by spaced training is determined by an association between the VDS and the context, so we call this associative memory the "context-signal memory" (CSM). The CSM then entails an association between two visual memories, a memory of the context (CM) and a memory of the signal (SM), each of which can be acquired independently (Tomsic et al., 1993, 1998; Hermitte et al., 1999).

The escape behavior of the crab to the VDS was found to be accounted for by the activity of a small number of lobula giant neurons (LGs) (Oliva et al., 2007; Sztarker and Tomsic, 2008). A crucial finding was that the response of the LGs to the VDS strikingly reflects the behavioral changes observed during learning and during long-term memory (Tomsic et al., 2003).

Here, we investigate the relationship between the LGs and some major attributes of the CSM. The first two experiments were aimed at assessing the processing of information regarding 
the stimulus (i.e., the memory about the "what"), while the third experiment was intended to assess the role of the LGs in the storage of the visual context information (i.e., the memory about the "where"). Results indicate that fundamental attributes of the stimulus memory can be accounted for by the performance of the LG neurons, while the visual context memory cannot.

\section{Materials and Methods}

Animals. Animals were adult male C. granulatus crabs collected in the coastal inlets of San Clemente del Tuyú, Argentina, and transported to the laboratory, where they were maintained as described previously (Tomsic et al., 2003). Experiments were performed within the first 2 weeks after the animal's arrival, throughout the entire year. Each crab was used in only one experiment.

Visual stimulus and recording procedures. The general methods for behavioral and electrophysiological experiments have been described in detail previously (Tomsic et al., 2003). Briefly, they were performed inside a Faraday cage completely covered to prevent outside visual stimuli from reaching the animal. The VDS (Fig. $1 A$ ) consisted of the horizontal displacement of a black rectangular screen $(7 \times 25 \mathrm{~cm})$ located $25 \mathrm{~cm}$ above the crab. It was driven by a motor at an angular velocity of $82 \% \mathrm{~s}$ and was controlled from outside the cage. The motion cycle, which consisted of a $90^{\circ}$ clockwise and counterclockwise excursion from the starting position and back, was completed in $2.2 \mathrm{~s}$. Each recording trial lasted $9 \mathrm{~s}$ and was composed of two cycles separated by $2 \mathrm{~s}$.

Behavioral experiments were conducted in an actometer consisting of a bowl-shaped container with a steep concave wall covered to a depth of $0.5 \mathrm{~cm}$ with marine water. It was connected to a transducer device that converted the crab's locomotion into voltage changes. These signals were digitized and recorded in a computer. For intracellular recording, the crab was firmly held in an adjustable clamp that allowed free movements of the walking legs but reduced movements of the chelae. The eyestalks were cemented to the carapace at an angle of $\sim 70^{\circ}$ from the horizontal line. With a tangential cut, we removed a small piece of cuticle from the tip of the right eyestalk without damaging the ommatidial area. The glass microelectrode was then positioned and advanced through the opening. Microelectrodes (borosilicate glass; outer diameter, $1.2 \mathrm{~mm}$; inner diameter, $0.68 \mathrm{~mm}$ ) yielded tip resistances of $40-60 \mathrm{M} \Omega$ when filled with $3 \mathrm{M}$ $\mathrm{KCl}$. Experiments were all performed at membrane resting potential.

Experiments began after a black curtain was lowered in the front part of the Faraday cage and the animal remained visually undisturbed for 10 min. In electrophysiological experiments, the adaptation period began after one LG had been impaled. Such a neuron was usually encountered within a few minutes of probing. Only one neuron per animal was recorded.

Experimental protocol. The study was organized in three sections, each of which included behavioral and electrophysiological experiments. The first two sections were aimed at gathering information regarding the stimulus component of the CSM, whereas the third section was directed at investigating the contextual component. We applied the classic spaced training used for the formation of the crab's CSM, consisting of 15 trials presented once every $3 \mathrm{~min}$. In parallel with the acquisition of CSM, the repeated presentation of the VDS (a stressful stimulus) during training induces a transient opioid-mediated analgesia (Valeggia et al., 1989; Romano et al., 1991). In mice and rats, this kind of stress-induced analgesia increases immobility and, hence, impairs the animal performance in behavioral tests (Kelly, 1986; McLaughlin et al., 2003). In the crab, the analgesia induced during training with the VDS causes a general reduction of the behavioral performance to different stimuli, including actual painful stimuli such as an electrical shock (Valeggia et al., 1989). This stimulus-nonspecific reduction was found to be transient, lasting up to 90 min following training (Romano et al., 1991). Consequently, despite the fact that enduring memory attributes such as stimulus specificity are acquired during training, the induced analgesia prevents their disclosure in behavioral tests performed shortly after training (Romano et al., 1991). The transitory inability to display stimulus specificity could be confounded with generalization. In addition, recent experiments in mammals have shown that stimulus specificity can increase with time
A
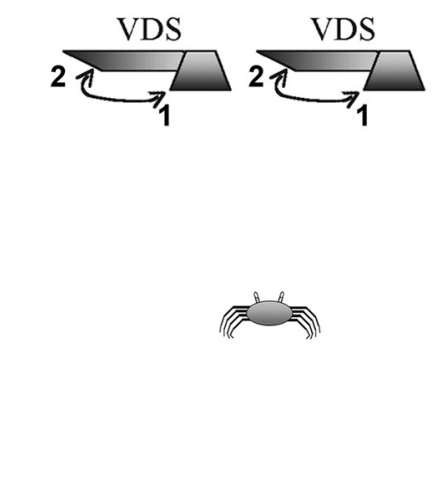

C
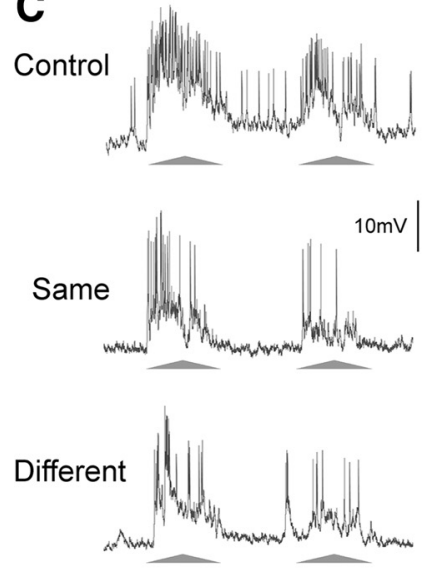

B Behavior

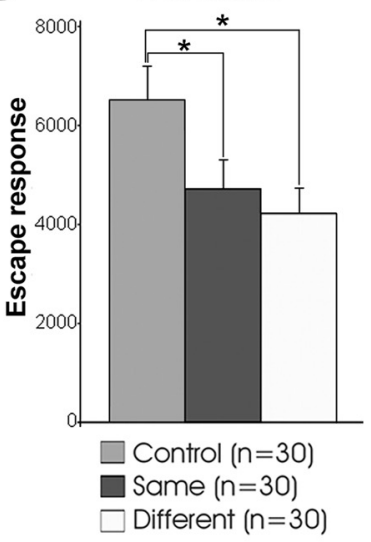

D Neuronal response

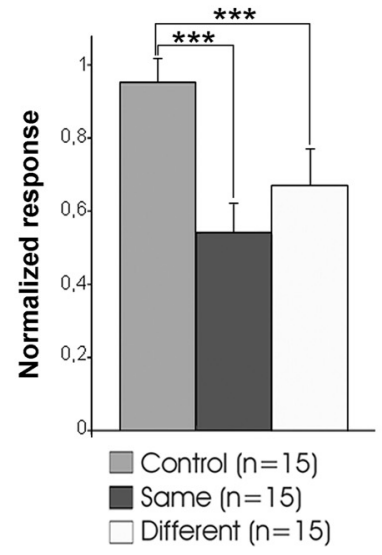

Figure 1. The crab's ability for generalization of stimulus position is supported by the LGs. $A$, Two VDSs located $25 \mathrm{~cm}$ above the crab were used independently to stimulate animals. The two VDSs were separated by $37 \mathrm{~cm}$, which represents an angular separation of $73^{\circ}$ for the crab. The VDS moved from 1 to 2 and back. Training consisted of 15 trials with fixed intertrial intervals of $3 \mathrm{~min}$. Behavioral experiments included a control and two trained groups, one tested with the same VDS as in the training and the other with a different one. $\boldsymbol{B}$, Mean escape responses of the three groups at a testing session $24 \mathrm{~h}$ after training. $\boldsymbol{C}$, Representative example of the responses of one LG to the VDS at the control trial (first training trial) and at test trials performed at the end of training with the same or the different VDS. The triangles below the traces represent the two cycles of stimulus movement comprising a trial (triangle base: $2.2 \mathrm{~s}$ ). $\boldsymbol{D}$, Mean neuronal responses of experiments as exemplified in $C$. Bars show mean $\pm \mathrm{SE}$. ${ }^{*} p<0.05 ;{ }^{* * *} p<0.005$. $\ln B, n$ represents the number of crabs in each group. $\ln D, n$ represents the number of neurons (one per animal) evaluated before training (control) and at the two test trials. See Materials and Methods for further details.

after training (Weinberger et al., 2009). For these reasons, in the present study the attributes of the behavioral memory were evaluated in test trials performed $24 \mathrm{~h}$ after training. The results obtained in these behavioral tests were then used to guide our search for the neural substrate that might reflect such memory abilities.

Even when some memory properties cannot properly be assessed through behavioral testing shortly after training, at the end of training there must be already changes in some neurons that provide support to their later behavioral expression. We previously found that changes in the response of the LGs measured right after training remain for at least $24 \mathrm{~h}$ and reflect the long-term behavioral expression of CSM (Tomsic et al., 2003). These results were obtained testing the animal's memory with exactly the same stimulus used to train them. By assessing the response of the LGs to slightly different stimuli immediately after training, in the first two series of experiments of this study we investigated whether the learning-induced changes that occurred in these neurons reflect the ability for stimulus generalization and stimulus recognition displayed by the animals $24 \mathrm{~h}$ after training. These experiments involved the use of two 
A
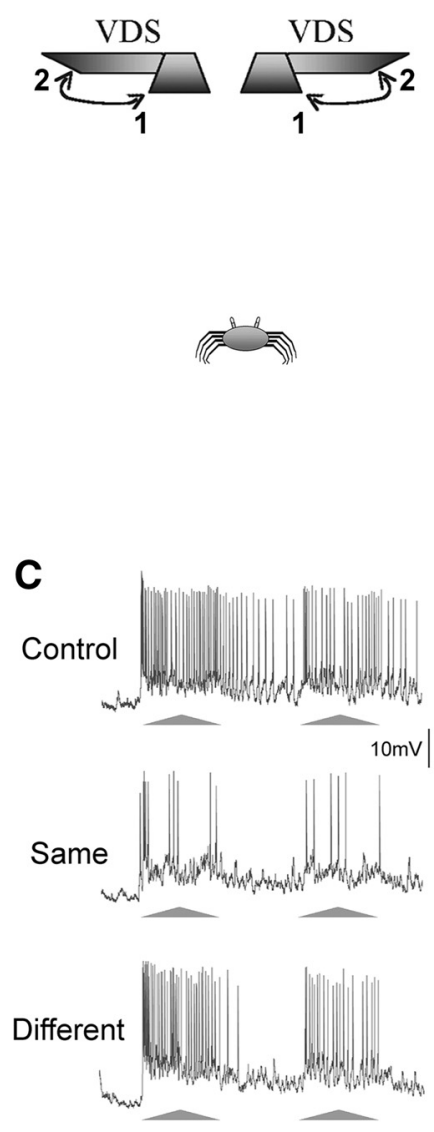

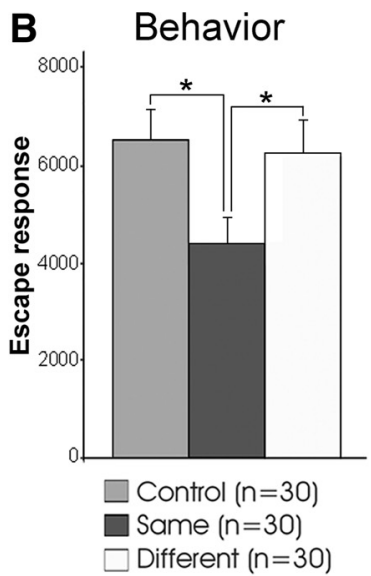

D Neuronal response

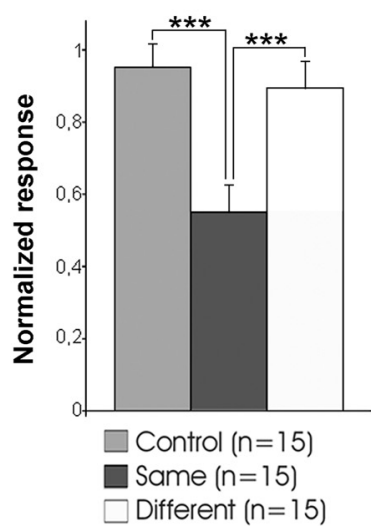

Figure 2. Stimulus-specific learning is accounted for by LGs. A, Two VDSs located above the crab with inverted motion cycles were used independently to stimulate crabs. They moved from 1 to 2 and back. Behavioral experiments included a control and two trained groups, one tested with the same VDS as in training and the other with a different VDS. $\boldsymbol{B}$, Mean escape responses of the three groups at the testing session. $C$, Representative example of responses of an $L G$ to the VDS at a control trial and at test trials performed with the same or the different VDS. D, Mean neuronal responses of experiments as exemplified in $C$. Further details and references are as in Figure 1.

stimuli (Figs. 1A, 2A). Behavioral experiments included the following three groups of animals: (1) those trained and tested with the same VDS (same group); (2) those trained with one VDS and tested with the other (different group); and (3) those exposed to context without training and tested with one of the two VDS (control group). In the electrophysiological experiments, neurons were recorded during training and at two tests performed 3 and 6 min after training. In classic experiments using a single VDS, responses obtained at these two testing times were not significantly different. In the present experiments, the first test was done with the same VDS used for training, and the second test with a different one. There are important variations in the number of VDS-evoked spikes among LGs recorded from different animals (Sztarker and Tomsic, 2004). Therefore, a baseline trial with each one of the two VDSs was performed $10 \mathrm{~min}$ before training and used to normalize the responses in subsequent trials. For each individual neuron, the baseline responses to one or the other VDS were very similar (Sztarker and Tomsic, 2004). A balanced number of crabs were trained with each VDS. Test responses were compared with that obtained at the first training trial (control).

The third section involved contextual changes between training and testing. Extensive research demonstrates that the crab's ability for memorizing the contextual environment is based on visual information (Tomsic et al., 1998; Hermitte el al., 1999) but not on other kinds of sensory information, such as ground texture, water level, water salinity, or water flavor (Frenkel et al., 2002; our unpublished results). Therefore, in the present study we used two different visual contexts created by

surrounding the actometer with a cylinder of striped or white walls (Fig. $3 A$ ). Methodologically, the electrophysiological experiments of the first two sections involved changes in the stimulus (same vs different) that could be done from a switch command located outside the Faraday cage without raising the curtain at the front of the cage. This allowed us to test the neuronal capacity for generalization and stimulus specificity right after training without distressing the animal. Conversely, the change of the contextual visual environment in the last experiment requires rearrangements within the cage that cannot be done without disturbing the animal and losing the impaled cell. Therefore, in this experiment the responses of the LGs were recorded $24 \mathrm{~h}$ after training. Unlike experiments 1 and 2, in this experiment the within-group neuronal variability could not be reduced by normalizing the data. Due to this and to the fact that the response of the LGs had to be evaluated in three separate experimental groups of crabs (control, same, and different), this experiment required recording from a larger number of neurons than the first two experiments. Both behavioral and electrophysiological experiments included the following three groups of animals: (1) trained and tested in the white context (same context group); (2) trained in the striped context and tested in the white one (different context group); and (3) exposed without training to either the white or the striped context and tested in the white one (control group). The responses of the LGs were recorded only at the testing session ( $24 \mathrm{~h}$ after training). Only one presentation of the VDS was used, and only one neuron per animal was recorded. Experiments were performed in a blind fashion, with the experimenter unaware of the treatment received by the animal on the training day.

Data analysis. The escape response was transduced and recorded as a train of voltage changes, with peaks corresponding to running steps of the escape reaction (Tomsic et al., 2003). Hence, the behavioral response in a trial was estimated by integrating the area of the voltage signals recorded during the periods of visual motion stimulation (cycles $1+2)$. The data are represented in an arbitrary scale. The neuronal response in a trial was estimated by the number of spikes recorded during the VDS stimulation (cycles $1+2)$. Data reported in the figures are mean \pm SEMs. Statistical analyses were performed with ANOVAs followed by LSD post hoc comparisons.

\section{Results}

\section{Generalization to new spatial positions}

Like humans, arthropods easily recognize learned objects over large changes in retinal position, a property commonly referred to as "position invariance" (Tang et al., 2004; Li et al., 2009). When a crab attempts to escape from the VDS inside the actometer, the stimulus position continuously changes over its retina. Even in these circumstances animals are able to acquire a strong memory, hinting at a rather flexible type of learning. But are crabs able to recognize the learned stimulus when it appears in a new spatial position $24 \mathrm{~h}$ after training? To test this possibility, we used two VDSs located in different positions above the crab (Fig. $1 A$ ). Animals were trained with one of the two VDSs and, 24 h later, they were tested with either the same or the different VDS. An equal number of crabs was trained with each one of the two VDSs. Results in Figure $1 B$ show that the responses of animals tested with the same or with the different VDS were similar, and both were significantly lower than the control group (memory expression is operationally defined by the statistical difference between a trained and an untrained control group). Therefore, $1 \mathrm{~d}$ after training crabs were able to transfer the learned response to a VDS located in a different position.

In Drosophila, a small group of neurons in the central complex that houses visual memory traces was proposed as a candidate to mediate position invariance (Liu et al., 2006). These neurons possess extensive parallel branches and are located in a brain region where visual information from both hemispheres converges, all of which make them suitable for the task. In the crab, 
these properties are already present in the optic lobes in the LGs. In fact, these neurons possess branches that extend horizontally throughout the retinotopic mosaic of the lobula (Fig. $3 C$ ), their receptive field extends over the entire crab visual field (Sztarker et al., 2005; Medan et al., 2007), and they process binocular information (Sztarker and Tomsic, 2004). Therefore, we investigated whether LGs can support the crab's ability to generalize the stimulus position. Neuronal responses were recorded throughout the training session with one of the two VDSs. Tests trials were performed with the same and then with the different VDS used for the training. Traces in Figure $1 C$ illustrate the responses of an LG neuron to the first VDS presentation at training and to the same and different VDSs in the test session. Figure $1 D$ shows the mean responses of 15 neurons. The LG responses to the same and the different VDSs were similar, and they were both significantly lower than the control values. This result shows that the LGs reflect the ability of crabs to generalize the learned stimulus into new spatial positions.

\section{Stimulus-specific memory}

Learning to reduce the escape response to a stimulus that proved to be harmless is advantageous, as it saves time and energy by preventing running away from riskfree stimuli. Being able to generalize the learned response to similar stimuli is also adaptive. However, animals need to keep reacting to unknown danger stimuli, requiring that the learning-induced reduction of the escape response should be stimulus specific. These two opposite arguments make the actual behavior a tradeoff between stimulus generalization and stimulus specificity. The final decision is based on the similarity between the new stimulus and the trained one. This similarity is evaluated along one or multiple perceptual dimensions that are relevant for each animal species. In the previous section, we showed that crabs are able to generalize when the new stimulus is presented in a different part of the visual space. Here, we investigate whether they are still able to generalize when an additional stimulus dimension, such as the sequence of motion direction, is altered. To test this, we used the two VDSs of the previous experiment, but inverted the motion cycle of one of them: starting from the center, each stimulus moved to one or to the other side of the crab (Fig. 2A). The experimental procedure was identical to the previous experiment. Results in Figure $2 B$ show that animals tested with the same VDS used on the training responded significantly less than control animals. On the contrary, animals tested with the different VDS responded like controls and significantly more than those tested with the same VDS. This indicates that crabs did not generalize between VDSs that differ in their sequence of motion. In this case, they showed a stimulus-specific memory.

We then investigated whether this stimulus-specific learning is reflected in the responses of the LGs. The electrophysiological
B Behavior

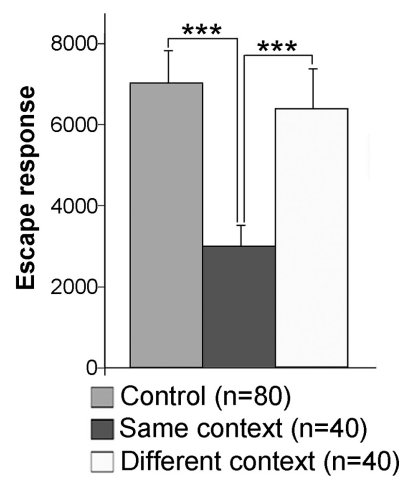

E Neuronal response

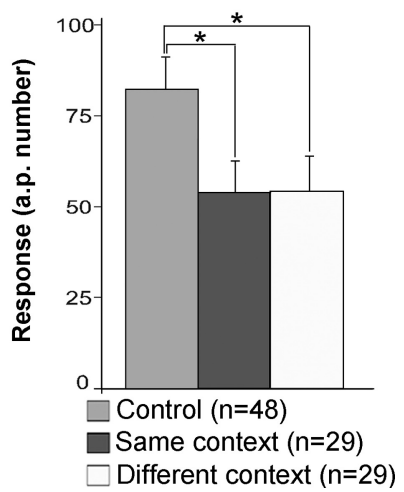

Figure 3. LGs house the trace for the signal but not for the visual context component of CSM. $A$, The two visual contexts used for
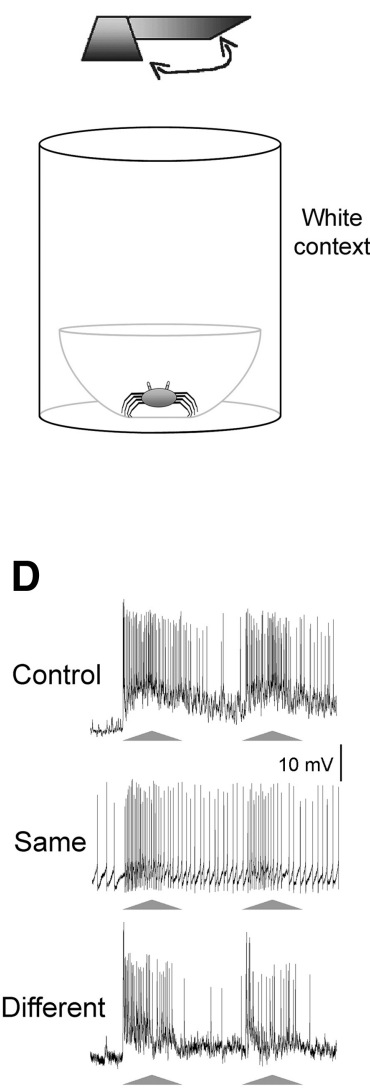

D

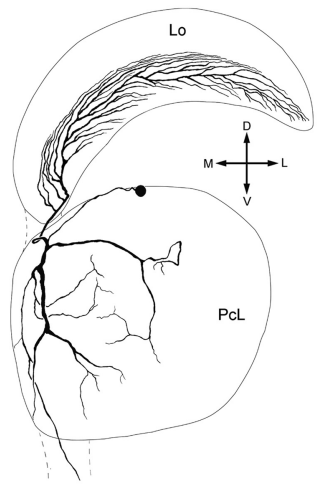
roups, one tested in the same context used during training and the other in a different context. Both behavioral and electrophys-

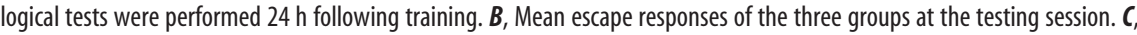

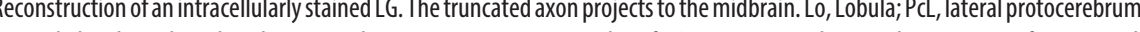
f crabs in each group. In $\boldsymbol{E}, n$ represents the number of neurons (one per animal) in each group. Other references are as in Figure 1. See Materials and Methods for further details.

procedures used were the same as those cited in the previous section. Traces in Figure $2 C$ illustrate the responses of an LG to the first VDS presentation of a training session and, after training, to the same or to the different VDS. Figure $2 D$ shows the mean responses of 15 LGs recorded in different animals. As expected, when tested with the same VDS used for training the response of the LGs was significantly lower than the control values. On the contrary, the response to the different VDS was similar to the controls, and significantly stronger than that obtained with the training stimulus. Therefore, LGs reflect the crab's stimulus specificity (i.e., they can distinguish a trained stimulus from a similar but unlearned stimulus).

\section{Context and signal memories}

As already mentioned, the CSM can only be evoked in the training context, implying an association between CM and SM. It has been shown that these two components can be acquired independently: CM by allowing the animal to simply explore a new context (Tomsic et al., 1993, 1998); and context-independent SM by performing a massed training (intertrial interval: $2 \mathrm{~s}$ ) with the VDS (Hermitte et al., 1999). Therefore, animals seem to store two visual components of the learned experience, one related to the stimulus (SM) and one related to the context (CM). Until now, 
the response of the LGs from trained crabs was assessed by keeping the contextual environment constant between training and testing. Hence, an important question that remained is whether the effect of changing the context in the testing session would be reflected by the performance of the LGs. In other words, do these neurons store the entire CSM trace or only the SM component? To test this, we evaluated the response of LGs to the VDS in animals located in the same or in a different visual context compared with where the memory had been acquired (Fig. 3A). Figure $3 B$ shows that $24 \mathrm{~h}$ after training crabs tested in the same context were able to express CSM, but those tested in a different visual context were not. Confirming previous results, the response of the LGs from animals tested in the training context was significantly lower than that in controls. The crucial result is that the LGs from animals that were tested in the different context also showed a response that was significantly lower than that of controls (Fig. 3D,E). These results show that whereas the learninginduced behavioral changes are context dependent, the neuronal changes induced in the LGs are independent of the visual context. In other words, the memory trace identified in the LGs only stands for the SM component of the CSM. Consequently, the traces corresponding to the contextual visual information and the context-signal association (the $\mathrm{CM}$ and the associative components of the CSM) must be stored in different places.

\section{Discussion}

Until recently, the predominant view on how arthropods learn and recognize visual patterns was that of a retinotopic template ("snapshot"), where the recognition of an object depended on the degree of overlapping between a memorized template and the perceived stimulus (Wehner, 1974). This theory, however, cannot account for flexible aspects of behavior such as the different types of generalization (Benard et al., 2006). In the present study, we show that crabs can achieve spatial generalization, which implies that they can recognize the learned stimulus independently of its retinal position (position-invariant object recognition). Li et al. (2009) proposed that, to do this, the visual system must transform the pixel-like image acquired by the retina into a neuronal representation that is unaffected by identity-preserving changes in the image such as those derived from variations in, for example, position, pose, size, and illumination. In primates, this transformation culminates in the inferior temporal cortex ( $\mathrm{Li}$ et al., 2009). In the crab, the anatomical and physiological properties of the LG neurons present in the optic lobes fulfill the requisites to perform this kind of neural computation (see Results). Our experiments show that the LGs reflect the ability of crabs to generalize the learned stimulus into new retinal positions. This means that LGs can hold a nonretinotopic representation of the learned stimulus that allows the identification of the VDS independently of its position. It is noteworthy that these results were obtained by using spaced training, which renders long-term memory. Conversely, an equal number of massed training trials renders only transient behavioral and neuronal changes (Tomsic et al., 2003), which are both retinotopic specific (our unpublished data). Thus, generalization, as well as true stimulus recognition, appears to emerge only with training protocols capable of generating enduring memory.

Generalization is less likely to occur when the mismatch between the learned stimulus and the new stimulus involves multiple dimensions (Benard et al., 2006). When we concurrently varied the position and the sequence of stimulus motion between training and testing, crabs were no longer able to generalize (Fig. 2). This means they recognized the new stimulus as being differ- ent from the learned one. We found that the LGs are capable of mediating this kind of object discrimination. Together, the results show that the LGs are involved in processing different attributes that allow the animal to remember the stimulus identity. These neurons then seem to house the memory trace related to what the learned stimulus is (stimulus recognition). On the other hand, the LGs are not involved in processing contextual visual information since their response was not affected by the contextual changes that prevent the retrieval of the CSM in the crabs (Fig. 3). We cannot exclude that contextual information other than visual, which might be relevant for the memory formation, could be processed by the LGs. However, we have never found an effect for nonvisual contextual changes on the expression of the crab's memory (see Materials and Methods). We can therefore conclude that the LGs do not hold the memory trace related to where the stimulus was learned (context recognition).

In the present study, the behavioral ability for remembering the attributes of the learned stimulus was evaluated $24 \mathrm{~h}$ after training, while the capacity of the neurons to reflect such ability was assessed immediately following training. Reasons for adopting this experimental design were explained in Materials and Methods. Because of this design, we cannot be certain that the performance of LGs would also match the behavior when measured $24 \mathrm{~h}$ after training. However, there is evidence supporting this view. Our previous studies show that spaced training induces immediate changes in the response of the LGs that last for at least $24 \mathrm{~h}$ (Tomsic et al., 2003). Thus, it is tenable to think that the reduced response of the LGs to the stimulus presented in a different spatial position recorded just after training (Fig. $1 D$, white bar) will remain reduced for $24 \mathrm{~h}$. Similarly, it is reasonable to expect that the high response of the LGs to the different stimulus (Fig. 2, white bar) will remain high on the following day. To confirm this, however, further experiments are required. At present, we can conclude that immediately after training the performance of the LGs already reflects the enduring ability of crabs to generalize the learned stimulus into new space positions and to distinguish it from a similar but novel one.

A remarkable outcome of these experiments is that immediately after training the response of the LGs allows prediction of the long-term response of the animals in a way that could not be predicted by assessing the behavioral performance. In fact, originally we performed these experiments at both behavioral and neurophysiological levels with testing sessions performed $3 \mathrm{~min}$ after training. The LGs reflected a capacity for generalization in the stimuli configuration of experiment 1 but not in that of experiment 2 (Figs. $1 D, 2 D$ ). At variance, the behavioral responses (data not shown) to the training stimulus and to the other stimulus were equally low in both test configurations. As explained before, this is because, shortly after training, the stimulusnonspecific effect caused by stress-induced analgesia (see Materials and Methods) precludes the crab from exhibiting stimulus specificity, thus giving the appearance of a capacity for generalization that extends even to stimuli of different sensory modalities (Valeggia et al., 1989; Romano et al., 1991). The fact that in the second experiment the response of the LGs to the different stimulus was identical to that of the controls, and significantly stronger than that obtained with the training stimulus (Fig. $2 D$, white bar), indicates that the stress-induced analgesia that transitorily affects the behavioral performance acts downstream of the LGs. This is further supported by the fact that the analgesia induced during training also affects the escape performance to an electrical shock (i.e., to a stimulus that is not processed in the visual pathway) (Valeggia et al., 1989; Romano et al., 1991). At 
present, we do not know where in the crab's nervous system the opioids released by stress exert their analgesic effects, but given that they affect responses elicited by stimuli of different sensory modalities, their action is likely to occur at the level of multisensory integrative centers.

The waning of the escape response to repeated presentations of a VDS and the ability to distinguish this stimulus from a slightly different one, described in our laboratory studies with Chasmagnathus, has been recently found to be present in the natural environment of the fiddler crab Uca vomeris (Hemmi and Merkle, 2009). As with Chasmagnathus, fiddler crabs were found to reduce their escape response to the repeated approach of a dummy predator, and the behavioral change proved to be stimulus specific and not retinotopic. Given the ecological differences between the two species, it would be interesting to see whether other features of the CSM of Chasmagnathus, like the endurance of the behavioral change, the capacity for stimulus generalization, and the context-stimulus association, can also be identified in fiddler crabs.

In Drosophila, as in the crab, important aspects of visual memory traces seem to be confined to a relatively small number of neurons (Liu et al., 2006). Nevertheless, stimulus cues and context information appear to be processed in separate neural circuits (Liu et al., 1999, 2006). For example, mutant flies with miniature mushroom bodies showed similar abilities to normal flies in being able to learn a visual discrimination task but were unable to process contextual information (Liu et al., 1999). This and other experiments in insects point to the mushroom bodies as the candidate site for the processing of contextual information (Liu et al., 1999, 2006; Menzel and Giurfa, 2001). There are many functional similarities between the hemiellipsoid bodies of crustaceans and the mushroom bodies of insects (McKinzie et al., 2003). There is then a possibility that the hemiellipsoid bodies could be involved in processing contextual cues in crabs. Further experiments will be needed to establish whether this is correct.

The experiments in flies and crabs involved different memory paradigms, methodologies (mutant flies and GAL4/UAS expression system following learning vs in vivo intracellular recording and staining), and temporal memory phases (short term vs long term), yet the results lead to the same conclusion: the small brains of arthropods share the capacity of modular processing with the big brains of vertebrates.

\section{References}

Benard J, Stach S, Giurfa M (2006) Categorization of visual stimuli in the honeybee Apis mellifera. Anim Cogn 9:257-270.

Chittka L, Niven J (2009) Are bigger brains better? Curr Biol 19:R995-R1008.

Frenkel L, Freudenthal R, Romano A, Nahmod VE, Maldonado H, Delorenzi A (2002) Angiotensin II and the transcription factor Rel/NF-kappaB link environmental water shortage with memory improvement. Neuroscience 115:1079-1087.

Giurfa M, Eichmann B, Menzel R (1996) Symmetry perception in an insect. Nature 382:458-461.

Giurfa M, Zhang S, Jenett A, Menzel R, Srinivasan MV (2001) The concepts of "sameness" and "difference" in an insect. Nature 410:930-933.

Hemmi JM, Merkle T (2009) High stimulus specificity characterizes anti-predator habituation under natural conditions. Proc Biol Sci 276:4381-4388.

Hermitte G, Pedreira ME, Tomsic D, Maldonado H (1999) Context shift and protein synthesis inhibition disrupt long-term habituation after spaced, but not massed training in the crab Chasmagnathus. Neurobiol Learn Mem 71:34-49.

Kelly DD (1986) Stress-induced analgesia. In: Annals of the New York Academy of Science, Vol 467. New York: New York Academy of Science. Li N, Cox DD, Zoccolan D, DiCarlo JJ (2009) What response properties do individual neurons need to underlie position and clutter "invariant" object recognition? J Neurophysiol 102:360-376.

Liu G, Seiler H, Wen A, Zars T, Ito K, Wolf R, Heisenberg M, Liu L (2006) Distinct memory traces for two visual features in the Drosophila brain. Nature 439:551-556.

Liu L, Wolf R, Ernst R, Heisenberg M (1999) Context generalization in Drosophila visual learning requires the mushroom bodies. Nature 400:753-756.

Lozada M, Romano A, Maldonado H (1990) Long term habituation to a danger stimulus in the crab Chasmagnathus granulatus. Physiol Behav 47:35-41.

McKinzie ME, Benton JL, Beltz BS, Mellon D (2003) Parasol cells of the hemiellipsoid body in the crayfish Procambarus clarkii: dendritic branching patterns and functional implications. J Comp Neurol 462:168-179.

McLaughlin JP, Marton-Popovici M, Chavkin C (2003) Kappa opioid receptor antagonism and prodynorphin gene disruption block stressinduced behavioral responses. J Neurosci 23:5674-5683.

Medan V, Oliva D, Tomsic D (2007) Characterization of interneurons responsive to visual stimuli that elicit escape behaviors in the crab Chasmagnathus. J Neurophysiol 98:2414-2428.

Menzel R (2001) Searching for the memory trace in a mini-brain, the honeybee. Learn Mem 8:53-62.

Menzel R, Giurfa M (2001) Cognitive architecture of a mini-brain: the honeybee. Trends Cogn Sci 5:62-71.

Mizunami M, Weibrecht JM, Strausfeld NJ (1998) Mushroom bodies of the cockroach: their participation in place memory. J Comp Neurol 402:520-537.

Oliva D, Medan V, Tomsic D (2007) Escape behavior and neuronal responses to looming stimuli in the crab Chasmagnathus granulatus (Decapoda: Grapsidae). J Exp Biol 210:865-880.

Pedreira ME, Maldonado H (2003) Protein synthesis subserves reconsolidation or extintion depending on reminder duration. Neuron 38:863-869.

Pedreira ME, Pérez-Cuesta LM, Maldonado H (2002) Reactivation and reconsolidation of long-term memory in the crab Chasmagnathus: protein synthesis requirement and mediation by NMDA-type glutamatergic receptors. J Neurosci 22:8305-8311.

Romano A, Lozada M, Maldonado H (1991) Nonhabituation processes affect stimulus specificity of response habituation in the crab Chasmagnathus granulatus. Behav Neurosci 105:542-552.

Sztarker J, Tomsic D (2004) Binocular visual integration in the crustacean nervous system. J Comp Physiol A Neuroethol Sens Neural Behav Physiol 190:951-962.

Sztarker J, Tomsic D (2008) Neuronal correlates of the visually elicited escape response of the crab Chasmagnathus upon seasonal variations, stimuli changes and perceptual alterations. J Comp Physiol A Neuroethol Sens Neural Behav Physiol 194:587-596.

Sztarker J, Strausfeld NJ, Tomsic D (2005) Organization of the optic lobes that support motion detection in a semiterrestrial crab. J Comp Neurol 493:396-411.

Tang S, Wolf R, Xu S, Heisenberg M (2004) Visual pattern recognition in Drosophila is invariant for retinal position. Science 305:1020-1022.

Tibbetts EA, Dale J (2004) A socially enforced signal of quality in a paper wasp. Nature 432:218-222.

Tomsic D, Massoni V, Maldonado H (1993) Habituation to a danger stimulus in two semiterrestrial crabs. Ontogenic, ecological and opioid system correlates. J Comp Physiol A Neuroethol Sens Neural Behav Physiol 173:621-633.

Tomsic D, Pedreira ME, Romano A, Hermitte G, Maldonado H (1998) Context-US association as a determinant of long-term habituation in the crab Chasmagnathus. Anim Learn Behav 26:196-209.

Tomsic D, Berón de Astrada M, Sztarker J (2003) Identification of individual neurons reflecting short- and long-term visual memory in an arthropod. J Neurosci 23:8539-8546.

Valeggia C, Fernandez-Duque E, Maldonado H (1989) Danger stimulusinduced analgesia in the crab Chasmagnathus granulatus. Brain Res 481:304-308.

Wehner R (1974) Pattern recognition. In: The compound eye and vision of insects (Horridge GA, ed), pp 75-113. Oxford: Clarendon.

Weinberger NM, Miasnikov AA, Chen JC (2009) Sensory memory consolidation observed: Increased specificity of detail over days. Neurobiol Learn Mem 91:273-286. 